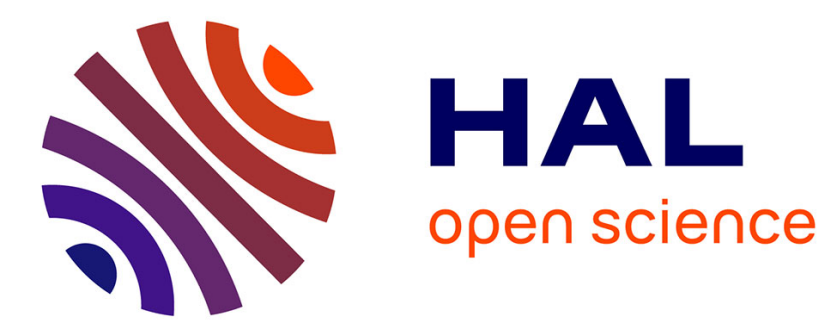

\title{
Experiential Hierarchies of Streets
}

Martin Tomko, Stephan Winter, Christophe Claramunt

\section{To cite this version:}

Martin Tomko, Stephan Winter, Christophe Claramunt. Experiential Hierarchies of Streets. Computers, Environment and Urban Systems, 2008, pp.41-52. 10.1016/j.compenvurbsys.2007.03.003 . hal-00771873

\section{HAL Id: hal-00771873 \\ https://hal.science/hal-00771873}

Submitted on 9 Jan 2013

HAL is a multi-disciplinary open access archive for the deposit and dissemination of scientific research documents, whether they are published or not. The documents may come from teaching and research institutions in France or abroad, or from public or private research centers.
L'archive ouverte pluridisciplinaire HAL, est destinée au dépôt et à la diffusion de documents scientifiques de niveau recherche, publiés ou non, émanant des établissements d'enseignement et de recherche français ou étrangers, des laboratoires publics ou privés. 


\title{
Experiential Hierarchies of Streets ${ }^{1}$
}

\author{
Martin Tomko ${ }^{\mathrm{a}}$, Stephan Winter ${ }^{*, \mathrm{~b}}$, Christophe Claramunt ${ }^{\mathrm{c}}$ \\ ${ }^{\text {a } C R C ~ f o r ~ S p a t i a l ~ I n f o r m a t i o n, ~ D e p a r t m e n t ~ o f ~ G e o m a t i c s, ~ U n i v e r s i t y ~ o f ~ M e l b o u r n e, ~}$ \\ Australia \\ ${ }^{\mathrm{b}}$ Department of Geomatics, University of Melbourne, Australia \\ ${ }^{\mathrm{c}}$ Naval Academy Research Institute, Brest, France
}

\begin{abstract}
Mental representations of spatial knowledge are organized hierarchically. Among people familiar with an urban environment, common spatial knowledge from these spatial mental representations enables successful communication of place and route descriptions, consisting of hierarchically-ordered references to prominent spatial features, such as streets. The more prominent a street is, the more likely it is to be known by the wayfinder receiving the directions. The automated construction of such descriptions therefore requires hierarchical data models ranking streets in street networks. This paper explores the reasons of overlaps in the content and hierarchical organization of common spatial knowledge among locals. We introduce a novel measure allowing to rank streets in a street network. This ranking allows to construct experiential hierarchies reflecting the shared experience of the streets in a city. The measure is derived from network connectivity measures, and takes into account the structure of the street network as well as the higher-order partition of the urban space into suburbs.
\end{abstract}

Key words: Experiential hierarchy, network analysis, centrality

\footnotetext{
* Corresponding author. Tel.: +61 38344 7875, Fax.: +61 393472916

Email addresses: m. tomko@pgrad. unimelb.edu . au (Martin Tomko), winter@unimelb.edu.au (Stephan Winter), claramunt@ecole-navale.fr (Christophe Claramunt).

1 The work has been supported by the Cooperative Research Centre for Spatial Information, whose activities are funded by the Australian Commonwealth's Cooperative Research Centres Programme. The authors would also like to acknowledge the valuable comments of three anonymous reviewers.
} 


\section{Introduction}

With repetitive interaction, people living in an urban environment get increasingly familiar with its layout (Siegel and White, 1975). With continuing interactions, the accuracy and completeness of this acquired knowledge increases. It is stored in mental representations that are to a large extent organized hierarchically (Hirtle and Jonides, 1985; Stevens and Coupe, 1978).

Despite individual distortions in mental representations (Couclelis et al., 1987), people familiar with the environment can successfully communicate spatial knowledge. This signifies that speakers are able to identify the part of their knowledge they expect to be shared with the hearer in a given communication context, and refer to it. Conversely, hearers are able to match the received information with their own mental representation.

The hierarchical organization of the spatial knowledge is reflected in hierarchical place and route descriptions. In hierarchical descriptions, references to elements of the city are introduced in order from most general to highly specific local references (Plumert et al., 2001; Shanon, 1979; Tomko and Winter, 2006b). Let us consider for example the route directions given to a taxi driver in Melbourne: "Take me to A'Beckett Street, off Elizabeth Street." (Fig 1). The speaker assumes that the knowledge of the highly prominent Elizabeth Street is common, without asking the driver first. The speaker also assumes that A'Beckett Street is not prominent enough to be localized by the taxi driver without reference to Elizabeth Street.

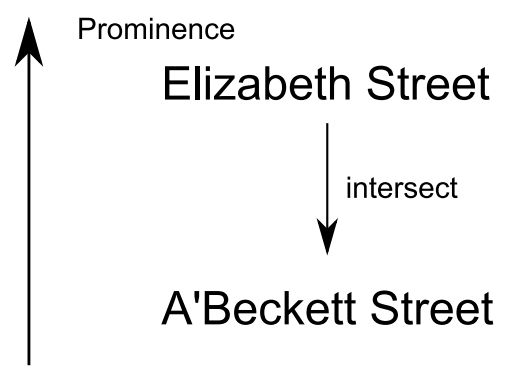

Fig. 1. Schema of hierarchical route directions.

In order to model and generate hierarchical place and route descriptions automatically, we need to characterize the prominent parts of the street network by quantifiable properties. In this paper, we explore the reasons of overlaps in the content and hierarchical organization of common spatial knowledge among locals. We assume that the emergence of the shared experience of the street network's hierarchical organization is due to the likelihood with which parts of it are used by locals.

Street connectivity influences the pattern of urban movement flows and determines the intensity of learning the urban layout. Frequently experienced parts of the network are prominent, and rank high in the hierarchical mental representations. Models considering the experiential significance of streets are currently not available. 
Administrative street hierarchies are traditionally the product of a classification grounded in design characteristics of streets (such as number of lanes), and not by their experiential prominence within the street network. Such hierarchies are therefore not necessarily suitable for cognitively ergonomic provision of route directions.

Our hypothesis is that the prominence of streets in the network relates to the spatial layout and properties of the street network and its spatial partition into suburbs. Our goal is the identification of a quantification measure reflecting the wayfinders' experience of the street network. This measure should consider connectivity at the street network level, as well as at higher levels of spatial organization, such as the suburb partition of the urban environment.

Structural analysis of the street network connectivity properties will be coupled by the analysis of its higher order functional partition. The result is a relative estimate of the prominence of a street in the city structure, presented as a ranking of the streets in a network in an experiential hierarchy.

An experiential hierarchy of streets allows the abstraction of the street network at different granularities, preserving the inherent logic of its structure. Experiential hierarchical models of spatial features, such as streets in the street network present a necessary input for hierarchical provision of wayfinding information to locals in hierarchical route directions (Tomko and Winter, 2006b).

The remainder of the paper is structured as follows: In Section 2, we point to previous work as the foundation on which we build our hypothesis and approach. We then continue our argument by identifying and testing network analysis measures for the reconstruction of experiential hierarchies in street networks (Section 3). Street networks are embedded in higher order partitions of the city into suburbs, and we consider this overlaid structure in the final hierarchical ranking of streets in experiential hierarchies in Section 4. The paper then concludes in Section 5.

\section{Background}

In this section we introduce the basic concepts in wayfinding and route direction research, relevance theory and topological analysis of city structures pertinent to our research. We introduce the concept of spatial hierarchies and show their importance in place and route descriptions. 


\subsection{Mental Representations and Hierarchical Spatial Reasoning}

People learn the spatial layout of their environment through repetitive interaction, such as wayfinding (Newman et al., forthcoming 2006; Siegel and White, 1975). The structure of their knowledge proceeds through stages of different characteristics. As people become familiar with the environment, they acquire survey knowledge and are able to locate and infer directions and distances between spatial features. In urban environments, these features have been categorized into elements of the city form (Lynch, 1960). In this paper, our attention is focused on the street network, consisting of streets, and the functional partition of the city into suburbs. Those would be classified by Lynch as paths and districts, respectively.

The accuracy and completeness of one's spatial knowledge increases with the continuing interaction. Individual mental representations are, however, always distorted through the individual experience of space and the cognitive processes leading to their construction. Individual responses to specific properties of the environment, cognitive capabilities and behavior in the environment are all highly individual.

People organize their spatial knowledge in hierarchically organized mental representations (Hirtle, 2003; Hirtle and Jonides, 1985; Stevens and Coupe, 1978). Hierarchies in mental representations are not likely to follow discrete hierarchical levels, but rather resemble to continuous order. The hierarchical organization of mental representations is reflected in spatial reasoning tasks, where dependence between the categorization of a specific spatial entity in the hierarchy and its use in the spatial task has been demonstrated (Plumert et al., 1995; Timpf and Kuhn, 2003; Timpf et al., 1992; Wiener and Mallot, 2003). Hierarchical mental conceptualizations emerge mostly for fast retrieval of approximate information. The results achieved using such information are acceptable if the information retrieved is sufficient to support the task of the agent, e.g. wayfinding.

\subsection{Hierarchical Organization in Street Networks}

At first sight, administrative hierarchies of urban networks seem to provide a classification of streets in the street network which may to some extent reflect the organization of the knowledge of locals. Administrative hierarchies are widely discussed in the literature (Eppell et al., 2001; Marshall, 2004). These hierarchical classifications are, however, the product of different needs and processes, as they primarily serve the function of urban traffic and transportation planners. Often the specification attributing a street to a specific hierarchical level is only vaguely stated and depends on the designer's decision.

Many researchers have pointed to the fact that hierarchical organization of anthro- 
pomorphic space is unnatural, claiming not only that boundaries may not be crisp, but also that the multitude of spatial connections allows for more than a single functional partition (Alexander, 1988). We argue, however, that due to the way in which humans acquire spatial knowledge, a hierarchy emerges in spatial systems where connections between elementary spaces are facilitated by transport networks. We do not claim that the individual hierarchies are identical among all individuals inhabiting the specific urban environment. These individuals, however, are able to relate information provided by others to their own knowledge, despite it being grounded in a different hierarchy. This is only possible to the extent to which the two hierarchies are similar, and we show how this similarity is due to the structural properties of the network as such, and its higher-order functional partition.

\subsection{Common Knowledge and Urban Structure}

Common knowledge is a term used by linguists to denote the knowledge which is held by multiple individuals in the communication act. If only two agents are concerned, the term shared knowledge is often used. Note that the elements of common knowledge are not explicit, i.e. the speaker does not necessarily know that the hearer has this knowledge as well. If this second-level knowledge can be established, i.e. by direct inclusion of a reference in an utterance, it is called mutual knowledge. While relevance theory questions the possibility of the existence of mutual knowledge (Sperber and Wilson, 1982, 1986), common knowledge plays an important part in communication and comprehension. In the communication of place descriptions and route directions, the speaker does not establish evidence of the existing spatial knowledge of the hearer. For example, the taxi passenger does not question the taxi driver about all the spatial features of the city she may know. Instead, the speaker assumes the taxi driver's knowledge of some of the spatial features in the given urban environment. This assumption is grounded in the way the speaker's own spatial knowledge was gathered. In this paper, we are specially looking at the role played by assumed common spatial knowledge in the communication of route directions.

The coarse structure of the city consists of spatial features known by the majority of locals. In hierarchical mental representations, this coarse structure fills the top levels. Hierarchical place or route descriptions will be understood by a hearer as long as they know the referents. As a consequence, the communication of hierarchic place and route descriptions can proceed only to a level of detail where referents are still part of the common knowledge. Once this level is reached, detailed turnby-turn descriptions are used. This switch is triggered by the hearer.

In communication of spatial information, features prominent due to their distinctness or salience are used as references (Michon and Denis, 2001; Raubal and Winter, 2002). This prominence can be due to visual, semantic and structural character- 
istics, as they have been identified for landmarks by Sorrows and Hirtle (1999). All three aspects are intertwined, but some of the elements of the city afford themselves to be more prominent in some of these aspects. In the case of streets, the experience of wayfinding through them makes the agents experience their structural role within the fabric of the city. Previous work on the salience of the elements of the city shows that the analysis of their structural properties allows to estimate their salience in the urban structure (Claramunt and Winter, accepted).

Central parts of the urban network are likely to be part of some trips of a wayfinder. The more different trips an individual performs in a given urban network, the higher is the likelihood that she experiences these parts of the network. More central parts of the network are experienced with higher frequency, and hence they are are better known (Tversky, 1993). They gain higher prominence in the mental representations of wayfinders.

Consciously or not, humans have the ability to assess the prominence of a part of the urban structure to others. Consequently, they refer to those parts in route directions, avoiding references to highly individual contexts, such as home and work locations. This objectivization is part of our effort to be collaborative in communication, i.e. to use references of high relevance to the hearer (Sperber and Wilson, 1986). The hearer expects relevant references, and interprets any reference in a manner maximizing its relevance, based on previous knowledge and the communication context.

\section{Experiential Hierarchies of Street Networks}

In this section, we define a method to reconstruct experiential hierarchies of streets in urban networks. Our selection of the appropriate measure for the quantification of the importance of the named street is motivated by its relation to wayfinding behavior. Structural properties of the street network elements are distinct, as they condition the visual or semantic experience. A street not visited will not be visually nor semantically prominent to a wayfinder. Furthermore, our approach is specific in its cognitive grounding, where structural and semantic parameters of the network elements are considered together, through the use of named streets. This approach complements previous structural approaches motivated by hierarchical spatial data generalization (Jiang and Claramunt, 2003).

\subsection{Basic Elements of the Network}

The selection of the elementary constituent of an urban network has an important impact on the results of many analyzes. Most urban analyzes use street segments as the building block of the street pattern. Urban planners introduced the concept 
of axial lines for urban space analysis (Hillier and Hanson, 1984). Our approach builds on the selection of a named street as the building block of the network (Jiang and Claramunt, 2004). Named streets are defined as the set of segments of the street network sharing the same street name. A street name is often the only characteristic of a street that is part of common knowledge. It's use in route directions points to the perception of the street as one feature, not a set of individual segments. Thus, named streets allow an integration of the semantic properties of the street network, supporting our main motivation is the construction of hierarchical data models for route descriptions.

It is possible that further concatenation of streets in mental representations takes place. For example, approaches based on strokes or continuity lines could also be used to provide coarser views on the urban network, for instance by amalgamation of streets changing their type, but not the name (e.g. Victoria street changing into Victoria parade) (Figueiredo and Amorim, 2005; Thomson and Richardson, 1999). Thus, local specifics of the street network need to be considered on a case-by-case basis.

The structural properties of the street network are further influenced by its functional embedding in the partition of the city into suburbs. Suburbs are parts of the city labeled with names, frequently used as references in place descriptions. Their precise boundaries may not always be known to the wayfinders, but their rough location is part of the common knowledge of the inhabitants of the city.

The experiential formation of hierarchies in mental representations is largely influenced by the structural properties of the urban network and the overlaid functional partition of the city into suburbs. Common network analysis in geographic information systems largely relies on network metrics, with networks represented as graphs where junctions are represented by nodes and streets by edges. We call such representations primal graphs. Space syntax theory uses dual graph representations of urban networks where nodes represent axial lines and their connections to adjacent axial lines are represented by edges (Hillier and Hanson, 1984). In this paper, the nodes in dual graph representations stand for named streets in the street network, or suburbs in the suburb partition of the city. We explore measures of network connectivity and derive one that quantifies and hierarchically ranks the streets in a network in a manner that is cognitively plausible.

\subsection{Measures for Experiential Network Hierarchies}

Network analysis provides a variety of centrality indexes, also applied to, or adapted for, urban analysis (Bera and Claramunt, 2003; Claramunt and Winter, accepted; Hillier and Hanson, 1984; Porta et al., 2006).

Degree centrality, in space syntax called connectivity, is a measure specifying the 
number of direct neighbors of a node in a network. It is a local measure. Thus, outside of the confined area of a suburb, degree centrality does not provide a measure of prominence.

Closeness centrality is a measure reflecting the average length of the shortest paths to all other nodes of the graph. Nodes or segments with high closeness centrality have low average length of the path to all other nodes in the graph. When applied to a given urban network, this measure reflects well the global structure of the city, revealing its core. In space syntax this measure is known as global integration, or relative asymmetry, and is usually applied on dual graphs constructed from axial lines (Hillier and Hanson, 1984). At first, it seems to be a plausible measure for the experiential hierarchical ranking of the urban network. Yet, in naturally evolved spatial transport networks, closeness centrality does not provide a reliable measure of hierarchical importance of a network element in the overall network. It distorts the hierarchy by assigning higher values to the streets in the core of the network. Side-lanes and alley-ways centrally located at the geographic center of the area of interest will always get high closeness values, as long as they create loops and thus do not lie on the periphery of the graph. This structural property, however, does not necessarily make them prominent.

A localized measure of integration considering only the network within a range of the three closest neighbors is often used to reveal the variation of integration across the network. This step-distance is based on empirical findings related to the average length of pedestrian walks. Its relevance to the formation of experiential hierarchies if other means of transport are considered is questionable. Furthermore, it is a measure designed to be applied to axial map analysis, as opposed to named streets.

Betweenness centrality provides the means to quantify the likelihood a graph node will lie on a shortest path between two other nodes of the graph. Betweenness centrality provides a global value for a specific network element. The hierarchy created by experience needs to take into account the likelihood of the usage of a specific street, not only its central aspect. Betweenness centrality is expected to reflect the probability of being selected by a frequent wayfinder. Hence the frequent use of the term choice. With the increasing number of trips performed by a wayfinder in a city, the likelihood that betweenness approximates the agent's experience of the urban environment increases. We will further investigate betweenness centrality and its derivatives as plausible measures for explaining the experiential hierarchy of urban networks. 


\subsection{Distribution of Centrality Values in Street Networks}

Let $G(V, E)$ denote a Graph $G$ consisting of vertices $V$ connected in pairs by edges $E$. Let $i, j$ and $k$ be distinct vertices of $G$. Let $n_{j k}$ denote the number of shortest paths between $j$ and $k$, and $n_{j k}(i)$ the number of such paths leading through $i$. Betweenness centrality $C_{i}^{B}$ of the vertex $i$ was defined by Freeman (1977) in $G(V, E)$ as follows:

$$
C_{i}^{B}=\sum_{i \neq j \neq k} n_{j k}(i) / n_{j k}
$$

We will study the distribution of betweenness centrality over several patterns of urban networks to reveal their hierarchical structure.

\subsubsection{Regular Grid Street Patterns}

Rectangular grid patterns consist of perpendicular streets forming blocks. All junctions have the same degree and are thus identical in their local structure. Such urban layouts are typical for modern planned cities. Downtown areas of major US and Australian cities follow the pattern, as well as some European planned cities, e.g. Barcelona. Some of these cities have a few streets intersecting the grid pattern diagonally. Such streets are usually well known. Betweenness centrality can reveal the relative importance of these streets, as we show by comparison with the remaining centrality measures.

Figure 2 presents a grid pattern and its dual graph representation consisting of 6 orthogonal streets, forming a grid of $2 \times 2$ blocks. The dual graph analysis was performed using the software Pajek (Batagelj and Mrvar, 2006). The graphs reveal the bipartite structure of the north-south and east-west streets. The betweenness values of the network of named streets are all equal. Such a network does not reveal any structural difference in any of the measures-degree, closeness or betweenness centrality, if we consider named streets as the building element of the network. If the element of analysis is a street segment, higher betweenness and closeness centrality values are attributed to the central part of the grid. As all the streets are intersected by the same number of connecting streets, degree centrality remains uniform in the whole grid.

In such a regular grid, however, the hierarchical ranking of streets by betweenness and closeness is identical. Hence, irregularities in urban layouts that impact on the perception of the city as such also cause variance between the centrality values. The addition of a diagonal street (node $N^{o} 7$ in Figure 3 ) in the grid network leads to a change of betweenness values of the streets. The north-south streets intersect all of the streets in the network and thus lie on most shortest paths. They top the ranking 


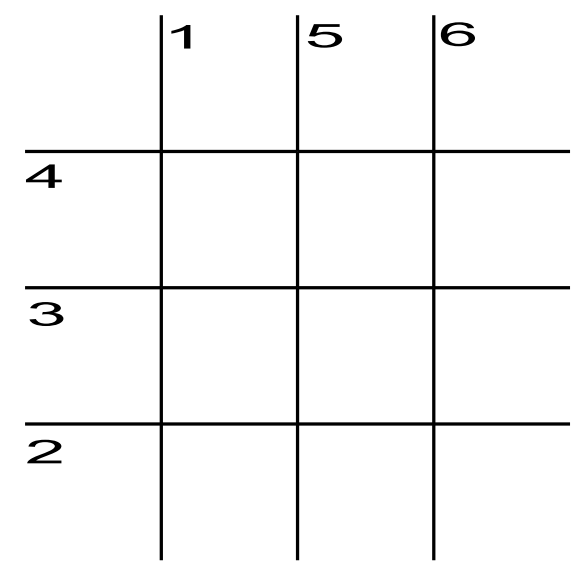

(a) Street network (primal graph)

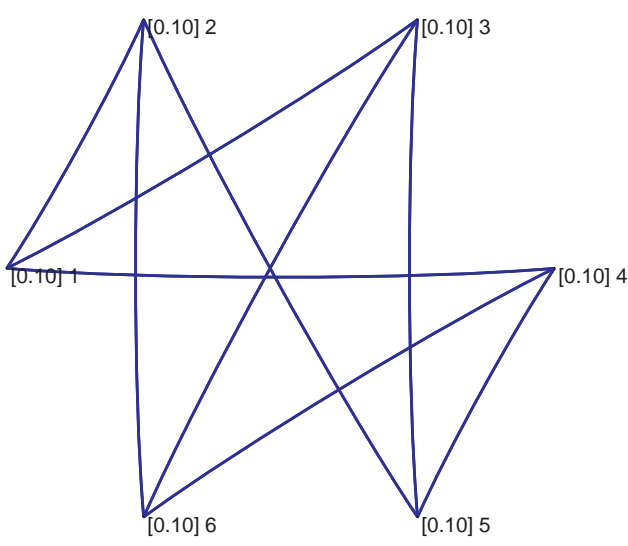

(b) Street network (dual graph). Labels represent betweenness values of streets.

Fig. 2. Graph representations of a grid network of named streets.

when ordered by betweenness, with the newly introduced shortcut following in the ranking. The ranking by degree and closeness centrality, however, does overemphasize the prominence of the shortcut, ranking the shortcut first. These measures therefore do not reflect the experience of the urban structure appropriately.

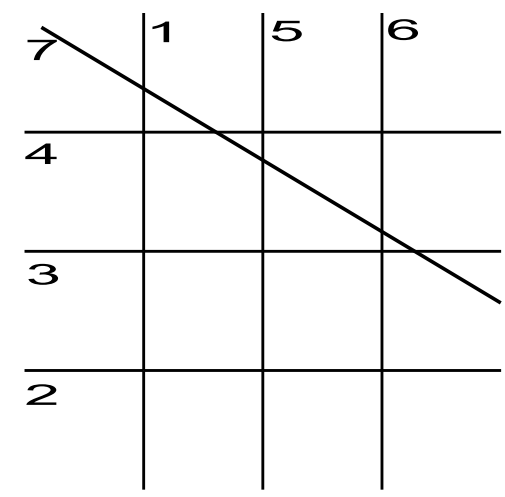

(a) Street network (primal graph)

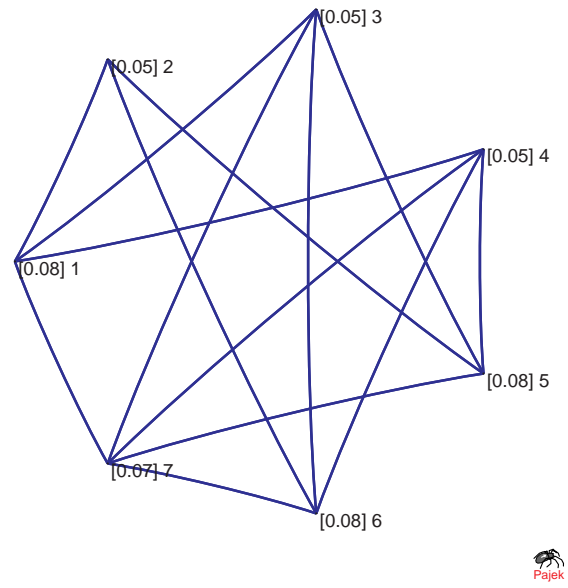

(b) Street network (dual graph). Labels represent betweenness values of streets.

Fig. 3. Graph representations of named streets grid with a diagonal street—shortcut.

\subsubsection{Star-like Street Patterns}

In a star-like network (Figure 4), the insertion of a shortcut changes the reachability of the peripheries of the street network involved. Betweenness centrality reflects 
this change in a manner that is consistent with the hypothesis of experiential formation of hierarchies in mental representations.

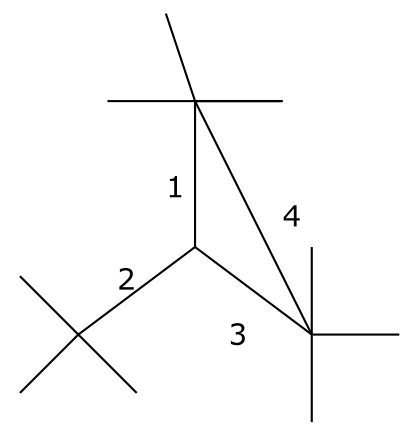

Fig. 4. A primal graph representation of a star-shaped street network with streets 1, 2, 3 and 4 labeled. Street 4 forms a shortcut.

It is the occurrence of shortcuts between internally highly connected subgraphs that motivates the use of betweenness for the reconstruction of the hierarchical structure of the street network. In a street network, such subgraphs may stand for distinct communities or suburbs, where the internal connectivity of the street sub-network is higher than that in the remainder of the city.
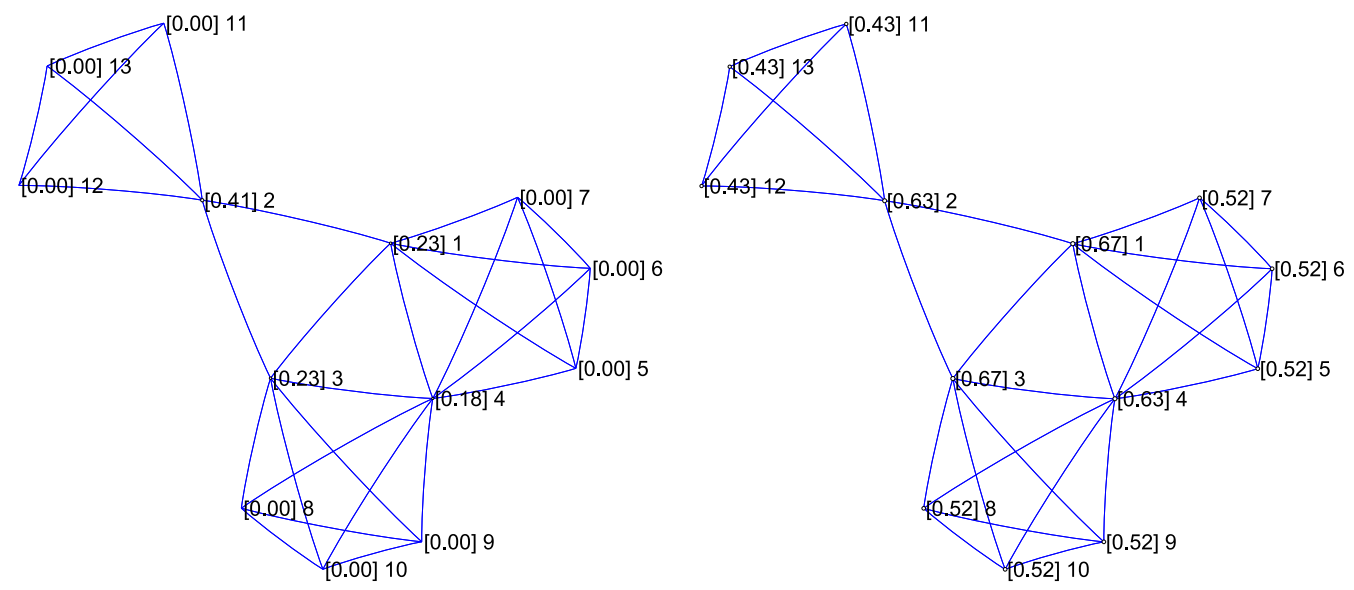

(a) Dual graph of the street network, with (b) Dual graph of the street network, with betweenness centrality values. closeness centrality values.

Fig. 5. Dual graph representations of streets in a star-shaped street network with added shortcut (street 4).

The dual graph representation of the street network shown as a primal graph on Figure 4 is shown on Figure 5. It reveals the structural changes caused by the shortcut - the addition of street 4 . The two distant peripheric parts of the network become directly connected. A new urban core is created by the triangle 1, 3 and 4 . The individual importance of streets 1 and 3 decreased with the introduction of the street 4 . The measure of betweenness reveals the alteration of experiential prominence of streets in the network. It preserves the high prominence of street 2 and reflects the lowering of the importance of streets 1 and 3 . Before the insertion of 
street 4, betweenness values for the streets 1,2 and 3 in the network were equal, 0.44 . After the insertion of street 4 , street 1 has a betweenness centrality value of 0.41 , streets 1 and 3 have values of 0.23 and street 4 a value of 0.18 . The ranking by betweenness thus reveals their structural importance in the street network appropriately.

Closeness centrality fails to reveal the alteration of this street network appropriately. The closeness centrality value of street 2 decreases as street 4 is inserted. Street 2, however, remains the only means of access to a significant proportion of the graph. Also, the relatively high values of closeness of the streets on the peripheries of the graph compared to the central streets have little justification from the experiential point of view. Thus, measure of closeness centrality fails to reveal the relative importance of the streets to the overall structure of the city. The importance of streets 2 and 4 would become even more prominent if a partition of the network into a suburb was introduced. We could cluster the peripheral parts of the graphs as suburbs. In such a case, the significance of streets 2 and 4 as links in the functional structure of the city would be emphasized even more.

\subsection{Experiential Street Hierarchies and the Suburb Structure}

The structural role of the streets facilitating movement between the functional partition of the city into suburbs should be considered for a refined ranking of streets in experiential hierarchies. Note that suburbs are chosen as the elementary modeling element due to their structural and semantic properties similar to the named streets. Suburb names are part of the common knowledge of the urban structure and represent a labeling system locals can rely on. To reflect the experiential delineation of suburbs inconsistent with administrative spatial partitions, other models of reconstructing the lynchean districts may be explored (Dalton, 2006).

We start from the assumption that the hierarchical importance of a street is relatively higher if it provides the only access to a suburb. It is, however, more common to have several streets with similar betweenness centrality values that connect the same suburbs. In turn, their respective betweenness values are lower, as they lie on alternative access routes. This is frequently the case in modern agglomerations with regular grid patterns in the center of the city. Those streets, however, are still very prominent, as their prominence is in turn supplemented by their membership in a structurally important suburb. As illustrated by Figure. 6, the two streets present alternatives for travel between the two nodes. Their respective betweenness centrality values would, in a network, be equal to a half of the value if a single street connected the two nodes. These streets, however, connect a central suburb, which may change the perception of their prominence in a city.

The higher-order structural embedding of a street contributes to its hierarchical 


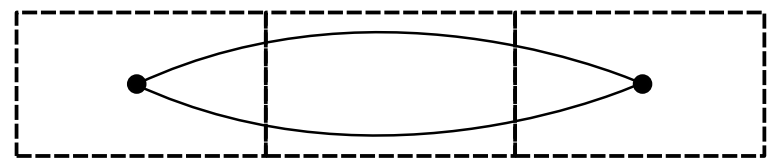

Fig. 6. Alternative streets with equal betweenness related to their suburb context (primal graph).

ranking in experiential hierarchies. The urban partition into suburbs thus represents an overlaying functional structure over the basic structure of the street network. Outside of the urban analysis literature, the relation between individual elements in a graph and their higher-order embedding was explored by Newman and Girvan (2004). To fine-tune the experiential hierarchy of streets, the structural relations between the suburbs should be explored.

In order to combine the functional and structural characteristics of the urban structure, we propose to consider the betweenness centrality of suburbs as the secondorder parameter influencing the ranking of streets in the network. A second graph is derived from the partition of suburbs where suburbs are the nodes of the graph, edges adjacency relationships between suburbs, as facilitated by streets. Betweenness centrality allows for consideration of the following structural properties in the street-suburb relationship as:

- Suburbs of high betweenness are crossed by a high proportion of possible trips in the network.

- Streets contained in suburbs of high betweenness are likely to be experienced more often.

Based on the betweenness centrality values of streets and suburbs, we introduce a novel measure for ranking of the streets in an experiential hierarchy of the street network. More formally, these notions are denoted as follows:

- Let $i$ denote a street in the street network;

- $D$ the set of suburbs in the city;

- $D^{i}$ the set of suburbs intersected by the street $i$;

- $d_{j}^{i}$ a suburb out of $D^{i}$;

- $C_{i}^{B}$ the betweenness centrality value of the street $i$;

- $C_{d_{j}^{i}}^{B}$ the betweenness centrality of the suburb $d_{j}^{i}$;

- $p_{d_{k} d_{l}}$ the number of shortest paths linking two suburbs $d_{k}$ and $d_{l}$ of $D$;

- $p_{d_{k} d_{l}}\left(d_{j}^{i}\right)$ the number of shortest paths linking two suburbs $d_{k}$ and $d_{l}$ that contains $d_{j}^{i}$.

We can then define $C_{d_{j}^{i}}^{B}$ as follows:

$$
C_{d_{j}^{i}}^{B}=\sum_{d_{j}^{i} \neq d_{k} \neq d_{l}} p_{d_{k} d_{l}}\left(d_{j}^{i}\right) / p_{d_{k} d_{l}}
$$


The experiential ranking value $E^{i}$ for a named street $i$ in the experiential hierarchy of the street network based on the betweenness centralities of the street network (Eq. 1) and the suburb partition of space (Eq. 2) is then defined as follows:

$$
E_{i}=\sum_{j} C_{i}^{B} \times C_{d_{j}^{i}}^{B}
$$

The value of $E_{i}$ is calculated based on the adjacency matrix of suburbs and streets. This matrix contains relations of suburbs intersected by streets in the street network. If such a relation exists, the betweenness centrality value of the street $i-C_{i}^{B}-$ is multiplied by the betweenness centrality value of the suburb $\left(d_{j}^{i}\right)$, and the resulting values are summarized. This can also be expressed in terms of a matrix product of street betweenness values and suburb betweenness values. $E_{i}$ is not a normalized value, and thus can be greater than 1 . The values of $E_{i}$ are calculated only for the purpose of ranking, their direct comparisons between different street networks are meaningless.

\section{Study of the Experiential Street Hierarchy of Melbourne}

\subsection{Betweenness Analysis of the Street Network of Melbourne}

We have performed an analysis of the urban pattern of the city of Melbourne, Australia, to test betweenness centrality distributions in the street network on a larger scale (Fig. 7). Melbourne is a relatively young city with a distinct regular grid pattern in its center (the Central Business District-CBD), and a system of streets which reach radially beyond the center.

The analysis of the urban structure was performed on a dataset consisting of named streets in the space syntax software Mindwalk (Figueiredo, 2002). The total number of streets in the analysis was 1175 . Mindwalk implements a computationally efficient version of betweenness centrality, called fast choice. It considers only one random shortest path between each pair of nodes in a graph, instead of generating all the alternative shortest paths. In larger networks, the differences in centrality values resulting from fast choice are statistically insignificant, which can be simply verified by multiple analysis of the same network.

The plot of the fast choice values of streets in the city of Melbourne is shown in Figure 8 , along with the visualization of the street network with streets of betweenness values of more than one $\sigma$ above mean value. Betweenness values range between 0.0017 and 0.2618 . The mean value calculated from the sample was $\bar{x}=0.0049$ with a standard deviation in the dataset $\sigma=0.0144$. There are in total 185 streets with betweenness values above the mean $\bar{x}$, and only 52 such that their betweenness 


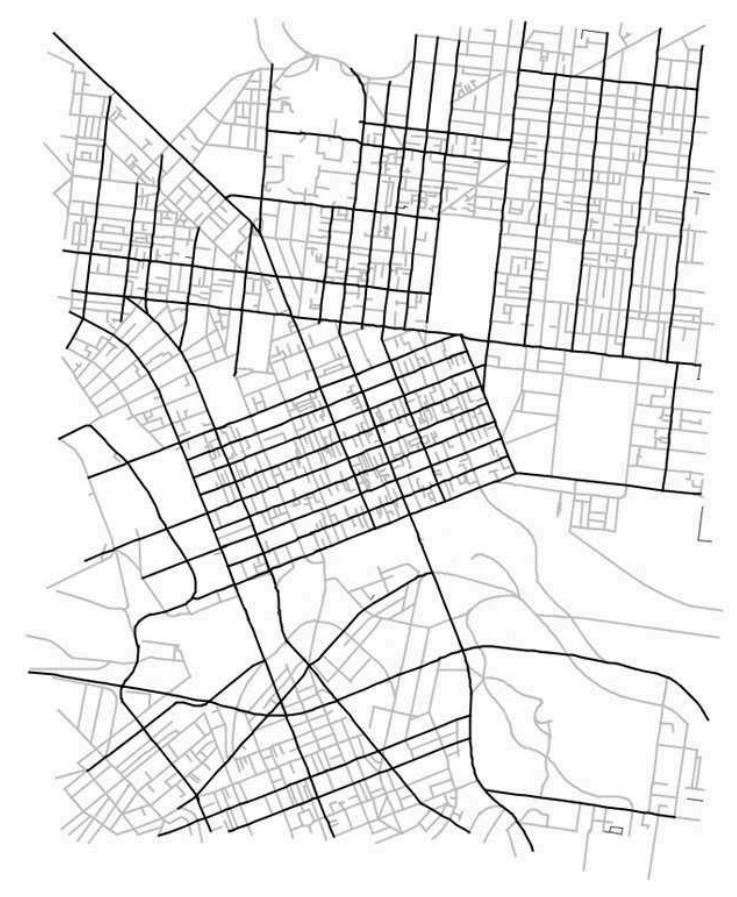

Fig. 7. Street network representations of streets of inner suburbs of Melbourne, with prominent streets highlighted (52 streets with fast choice values $\sigma$ above mean).

value is more than a $\sigma$ above $\bar{x}$. The distribution of betweenness values follows a power law, with the exponent $\alpha=3.37$ (calculated according to Newman (2005)).

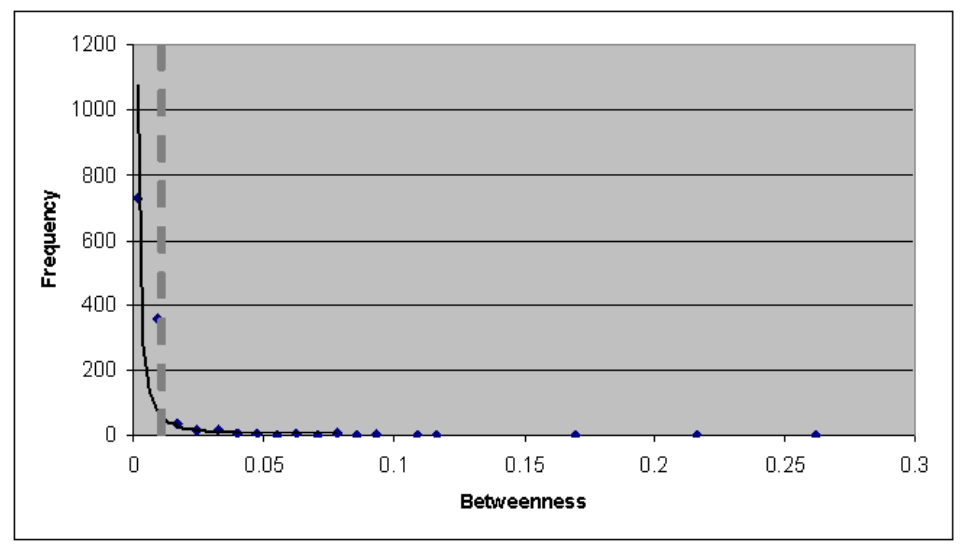

Fig. 8. Power-law distribution of named street betweenness values in the street network. The count of streets decreases radically with increasing betweenness, suggesting a hierarchy of streets in the network. The mean value is marked in dashed line.

Victoria Street, the named street of highest betweenness, has a betweenness value 154 times higher than the streets with the lowest value, and even the streets with the mean value are likely to appear on a shortest path within the network 3 times as often then the lowest ranking ones.

The streets with high betweenness centrality values (Table 1) correspond to the 
most prominent streets of Melbourne, well known to virtually all its inhabitants. Victoria Street is the major east-west street in the CBD, and has a similar role as King Street, which in turn channels most of the north-south traffic. All of the streets with high betweenness values are likely to be experienced by inhabitants of Melbourne, and are frequently used for travels to inner city suburbs. The experience of those streets is intensive and frequent. An exploration of the spatial distribution

Table 1

Hierarchy of most important streets in inner Melbourne.

\begin{tabular}{|c|l|c|}
\hline Rank & Street & Fast choice \\
\hline 1 & Victoria St. & 0.2618 \\
\hline 2 & King St. & 0.2121 \\
\hline 3 & Swanston St. & 0.1663 \\
\hline 4 & Nicholson St. & 0.1113 \\
\hline 5 & Spencer St. & 0.1018 \\
\hline
\end{tabular}

of streets with high betweenness values shows that they are relatively evenly distributed across the study area. This was expected, as betweenness centrality is not influenced by boundary effects. The distribution corresponds with the empirical experience where a relatively small proportion of streets in a city form a cognitively important skeleton structure (Kuipers, 2001). Thus, even a small set of all the streets in the street network may provide convenient referents for place and route descriptions.

This result illustrates well the plausibility with which betweenness centrality reveals the experiential hierarchy in an urban network, and also points to the importance of named streets as a conceptual building elements in the communication about the urban network. The network characteristics of the named streets-the concatenation of the street segments-as a basic network element are vastly different from those of the individual segments. The ranking shows how structural characteristics of these spatially chunked elements relates with their shared semantic properties—street names.

\subsection{Analysis of the Suburb Structure of Melbourne}

The functional partition of Melbourne into suburbs was analyzed by betweenness centrality. For this purpose, an adjacency matrix of suburbs has to be constructed, in which suburbs are noted as adjacent if connected by a named street. This relation between streets and suburbs can be found in natural language route expressions, such as: "... take Victoria Street to Richmond". We do not consider two suburbs adjacent if a street only touches but does not cross their boundary. 
The network of the 185 streets of Melbourne with betweenness centrality values above mean (Section 4.1) was used for the construction of the adjacency matrix. The high betweenness values of a few suburbs show their role as natural transit regions within the urban structure (Fig. 9).

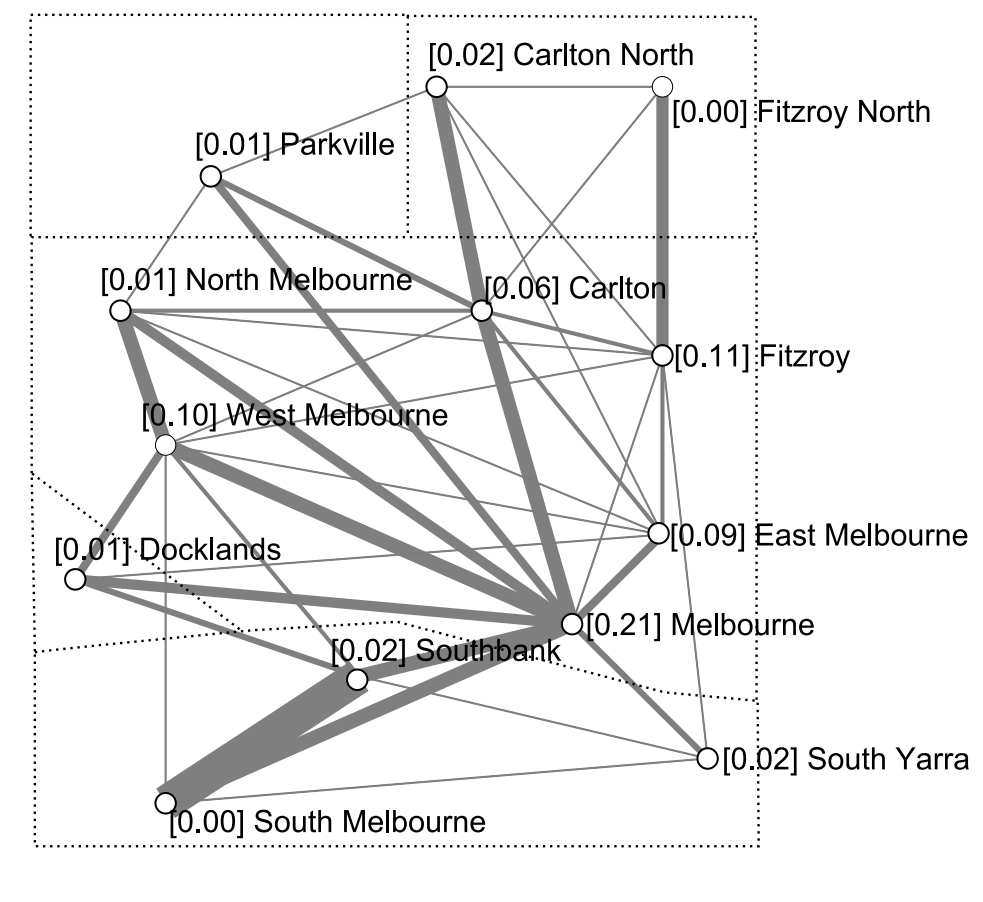

Fig. 9. Structure of inner suburbs of Melbourne. Betweenness of the suburbs is indicated by labels, cardinality of connections by line thickness. The clustering of the suburbs in higher-order regions is indicated by dotted lines.

In the selected study area, the configuration of the street network assures a high betweenness centrality value to the suburb of Melbourne (consisting of the postcode areas of the $\mathrm{CBD}$ and adjacent parklands). Its betweenness centrality value $C_{\text {melb }}^{B}$ is 0.21 . Most of the routes between the opposite parts of the city have to transit through it. Following in the ranking by betweenness are the suburbs of Fitzroy, West Melbourne, East Melbourne and Carlton, together creating the inner core of the city.

We performed a clustering analysis of the suburb structure of Melbourne in order to construct this higher-order structure based on the street network properties. This analysis allows us to verify whether there is a structural relation between the layout of the street network and the intuitive partition of the city into the central suburbs, the southern and northern suburbs divided by the Yarra River. The assumption is that prominent streets in the city structure should preserve their roles even at this coarser level of partition of the city.

The faction analysis algorithm provided by the software NetDraw (Borgatti, 2002) retrieves naturally cohesive groups of suburbs within this region (for an overview of the algorithm, see Hanneman and Riddle (2005). The optimal clustering coefficient 
was obtained for five factions, as shown on Figure 9. This clustering follows and intuitive division of the inner part of Melbourne into the central suburbs north of the Yarra River (Melbourne, West Melbourne, North Melbourne, Carlton, Fitzroy and East Melbourne), the part of the city on the south of the river (Southbank, South Yarra, South Melbourne) and the northern suburbs (Carlton North, Fitzroy North). The distinct region of Docklands represents a new development somehow isolated on the other side of a sports stadium, and is in this respect similar to Parkville, which consists mostly of parkland with only a few connections to the surrounding suburbs.

This analysis was performed to identify a hierarchically higher-order organization of the city. It has successfully identified the major north-south division of the city by the river, as well as the outer ring of inner suburbs around the CBD. This partition is strongly experienced by the inhabitants of Melbourne. As we can see, the structure of the suburb partition of the city is closely related to that of the street network. As such, both should be considered when constructing experiential street hierarchies.

\subsection{Experiential Hierarchy of the Street Network of Melbourne}

The results of betweenness analysis of the street network of Melbourne and of city's higher-order functional partition into suburbs serve as the inputs for the reconstruction of a refined experiential hierarchy of the street network by application of the experiential ranking $E_{i}$ (Eq. 3).

The refined ranking of the named streets of Melbourne by $E_{i}$ is shown in Table 2 .

Table 2

Hierarchy of most important streets in inner Melbourne (with consideration of suburb structure).

\begin{tabular}{|c|l|c|}
\hline Rank & Street & $E_{i}$ \\
\hline 1 & Swanston St. & 0.2176 \\
\hline 2 & Victoria St. & 0.1518 \\
\hline 3 & Elizabeth St. & 0.0658 \\
\hline 4 & King St. & 0.0658 \\
\hline 5 & Flinders St. & 0.0358 \\
\hline
\end{tabular}

The ranking shows changes compared to Table 1. It reflects well the structural and functional role played by the streets identified in the city of Melbourne. Swanston Street is the major north-south connector between the northern inner suburbs and the suburb of Melbourne, and serves its eastern part. Elizabeth Street serves a similar purpose, but runs more to the west. King Street is yet another north-south con- 
nector, but in this case connects the westernmost edge of city and the western inner suburbs with the southern suburbs on the other side of the Yarra River. This system of major connectors is completed by Victoria Street, which connects the east with the west on the northern side of the inner central suburbs. Its function is complementary to that of Flinders Street, serving the same purpose on the southern border of the CBD. When relating this ranking with the higher-order partition of the city (Section 4.2), the top-ranking streets of Melbourne are still identifiable as connectors between the higher-order clusters of suburbs (Fig. 10). As such, they represent a real backbone of the street network.

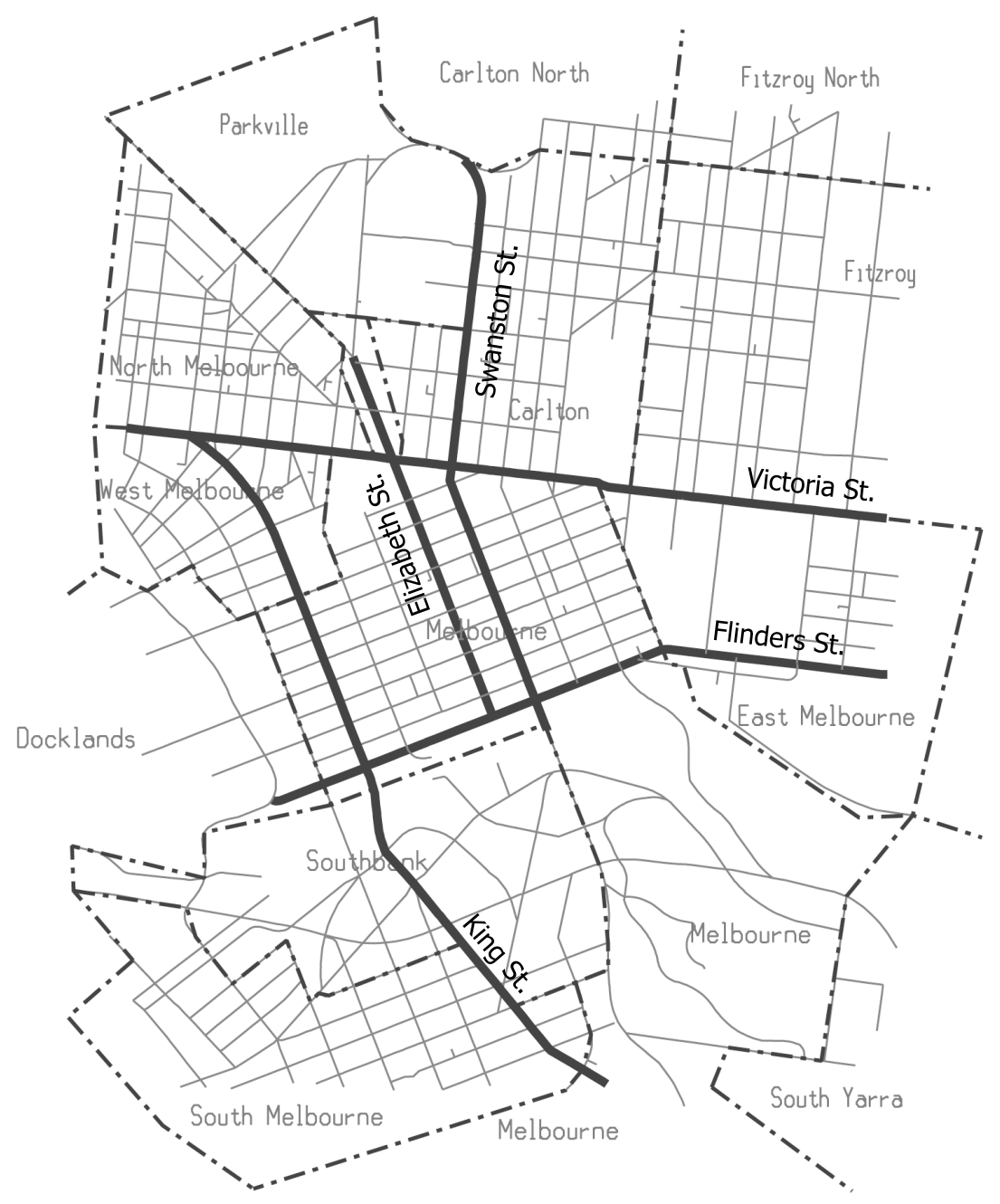

Fig. 10. The five major streets of Melbourne as identified by the combined street/suburb betweenness (bold). Outlines of the suburb boundaries in dashed stroke.

The perception of prominence may be reinforced by the fact that these streets are all multi-modal corridors (including tram lines) or are parts of the city bypass system. One can, however, argue that the presence of tram rails is a secondary result of an engineering process, by which the structurally significant function of these streets was acknowledged and reinforced. 
The joint consideration of the street network structure together with the suburb partition of the city allow for a reliable identification of the most prominent streets of the street network. We are now turning our attention to the integration of prominent streets as references in the communication of granular route directions.

\subsection{Further Considerations for Experiential Hierarchies of Street Networks}

The structural position of a street affects its use in the street network. The parts of the network with high betweenness centrality attract more traffic flow and business. Ultimately, the intensity of use leads to overloading of these parts of the network. Administrative measures, such as alteration of traffic conditions from a two-way into a one-way street, are often taken to alter the structure of the network in order to decrease the traffic. When the overcrowding exceeds some acceptable threshold, wayfinders optimize their trips. Locals with a thorough knowledge of their environment seek shortcuts and by-passes. The network properties of the alternative streets found also have relatively high values of betweenness, even if they may not lie directly on the shortest paths.

Non-structural factors also contribute to the perception of prominence of streets. Semantic and visual characteristics of other types of elements of the city, such as landmarks, have been widely studied before and we have no reason to believe that they do not influence our experience of the street network. Visual characteristics of shopping arcades and important pedestrian zones can be highly salient. The effect of semantic salience is harder to judge, but is certainly present as well. It is, however, unclear how these characteristics mix together to contribute to the overall prominence of a specific street. Furthermore, our effort is focused on the identification of prominent parts of a transportation network in order to support the communication between two agents. If only a minimal knowledge about the context of the recipient of the information is established, structural properties of the street network provide means to identify overlapping knowledge with a high degree of reliability, based on some common behavioral patterns which may be inferred.

\section{Conclusions}

We have introduced the notion of experiential hierarchies in mental representations of urban networks as a product of the interaction of wayfinders with streets in the city. The intensity of experience is a reflection of the functional and structural importance of a specific street in the urban network. Such experiential hierarchies represent one of the fundamental structures on which we can base our assumptions about the spatial knowledge of others. It allows for a selection of references to elements of a city in communication such as the exchange of route directions. 
We have discussed the effects of three basic measures of network centrality for the plausibility of best operationalization of the experiential hierarchies: degree centrality, closeness centrality and betweenness centrality. We argue for the application of betweenness centrality as a basis for the novel measure. Betweenness centrality reflects the hierarchical importance of streets in the city network, without being influenced by distortions due to the boundary effects. Our approach is based on the use of named streets, which provides a mean to analyze street networks reflecting additional aspects of the shared experience, namely the labeling of the streets. In the communication between two locals who are mutual strangers and thus have no additional shared context, this is likely to be one of the most important characteristics of streets one can rely upon and one that is expressed in the utterance. Thus, named streets are good candidate for references in automatically generated route directions.

We further illustrate with the central part of the city of Melbourne, Australia, how betweenness, or more precisely its computationally efficient implementation of fast choice, reveals the hierarchical structure of this urban network. The distribution of the betweenness values identifies a small amount of highly prominent streets.

We then propose to fine-tune this hierarchical ranking of streets in the network by considering the higher order embedding in the suburb partition of the city. We construct a graph representation of the connectivity of suburbs in the urban structure, and argue for the use of betweenness centrality values of suburbs as an additional factor influencing the prominence of streets connecting them. Experiential ranking $\left(E_{i}\right)$ is the novel measure proposed, merging the structural properties of the streets in the network with influences of the functional partition of the urban network into suburbs. The resulting ranking of streets follows our experience with the street network of Melbourne.

The empirical success of route direction communication in our daily lives suggests that there is a large overlap in the structures of our spatial knowledge, and that the knowledge of the prominent parts is common. Thus, a certain degree of difference in individual experiential hierarchies is not a barrier to reaching understanding. The match between the prominent parts of the hierarchies of the speaker and of the hearer is high when most of their elements are identical, but not necessarily ranked in the same order. Due to the power-law distribution of street prominence values in the experiential hierarchies, highly prominent streets have values higher by magnitudes than those of low prominence. This also allows the use of the proposed objectivized experiential hierarchy, based on the quantification of structural prominence of streets, for an automated construction of route directions by an automated service.

Individual experiential hierarchies are continuous rankings, and it is difficult to draw a line separating prominent and non-prominent streets. It is, however, possible to approximate this limit by the mean value in the distribution. The bulk of the 
streets in the hierarchy are below the mean value of prominence. In the case of the city of Melbourne, the streets around the mean value of prominence are about 3 times as prominent as those with the lowest prominence. Due to the even distribution across the city, only a few top ranking streets can be called prominent and used in hierarchical place and route descriptions for wayfinders with local knowledge of the city.

Urban datasets that are structured to match the experience of locals are an important input for improved communication of spatial information in context aware applications, such as route direction generation for locals. The reduction of the total length of such route directions, compared to traditional turn-based directions, or to directions using spatial chunks (Richter et al., 2004), is due to process of relevancebased selection of referents. The direction giver assumes shared knowledge of the coarse structure of the environment with the wayfinder, and describes the location of the target in a zooming manner. We have proposed a formal model to determine the referents in such granular route directions (Tomko and Winter, 2006a,b). This model was applied on hierarchical partitions of districts.

The reconstruction of the experiential hierarchy in the urban street network is necessary for a coherent assignment of hierarchical values to streets and suburbs. This will allow the development of an integrated hierarchical dataset of various elements of the city, allowing for the selection of the most relevant referents for granular route directions. Furthermore, it is also necessary to assess the internal structure of the street network within the individual suburbs. As hinted by Dalton (2006), it is possible that our experience of suburbs does not exactly match the administrative partition of the city, but is also strongly determined by the structure of the street network. This could improve the constructed experiential street hierarchies.

\section{References}

Alexander, C., 1988. A City is not a Tree. In: Thackara, J. (Ed.), Design After Modernism: Beyond the Object. Thames and Hudson, London, pp. 67-84.

Batagelj, V., Mrvar, A., 2006. Pajek-Program for Large Network Analysis, v1.12. http://vlado.fmf.uni-lj.si/pub/networks/pajek/.

Bera, R., Claramunt, C., 2003. Topology-Based Proximities in Spatial Systems. Journal of Geographical Systems 5 (4), 331-415.

Borgatti, S. P., 2002. NetDraw v. 2.043. Graph Visualization Software.

Claramunt, C., Winter, S., accepted. Structural Salience of Elements of the City. Environment and Planning B: Planning and Design .

Couclelis, H., Golledge, R. G., Gale, N., Tobler, W. ., 1987. Exploring the Anchorpoint Hypothesis of Spatial Cognition. Journal of Environmental Psychology 7, 99-122.

Dalton, N. S. C., 2006. Configuration and Neighborhood: Is Place Measurable? 
In: Space Syntax and Spatial Cognition Workshop of the Spatial Cognition '06 conference. Bremen, Germany.

Eppell, V., McClurg, B. A., Bunker, J. M., 2001. A Four Level Road Hierarchy for Network Planning and Management. In: Jaeger, Vicki (Eds.), 20th ARRB Conference. Melbourne, Australia.

Figueiredo, L., 2002. Mindwalk.

Figueiredo, L., Amorim, L., 2005. Continuity Lines in the Axial System. In: The Fifth Space Syntax International Symposium. Delft University of Technology, Delft, The Netherlands.

Freeman, L. C., 1977. A Set of Measures of Centrality Based on Betweenness. Sociometry 40 (1), 35-41.

Hanneman, R., Riddle, M., 2005. Introduction to Social Network Methods.

Hillier, B., Hanson, J., 1984. The Social Logic of Space. Cambridge University Press, Cambridge, UK.

Hirtle, S., 2003. Neighborhoods and Landmarks. In: Duckham, M., Goodchild, M., Worboys, M. (Eds.), Foundations of Geographic Information Science. Taylor \& Francis, London and New York, pp. 191-203.

Hirtle, S., Jonides, J., 1985. Evidence of Hierarchies in Cognitive Maps. Memory and Cognition 13, 208-217.

Jiang, B., Claramunt, C., 2003. A Structural Approach to the Model Generalisation of an Urban Street Network. Geoinformatica 8 (2), 151-171.

Jiang, B., Claramunt, C., 2004. Topological Analysis of Urban Street Networks. Environment and Planning B: Planning and Design 31, 151-162.

Kuipers, B., 2001. The Skeleton in the Cognitive Map. A Computational Hypothesis. In: 3rd International Space Syntax Symposium. Atlanta.

Lynch, K., 1960. The Image of the City. The MIT Press, Cambridge, Massachusetts, USA.

Marshall, S., 2004. Streets and Patterns. Spon Press.

Michon, P.-E., Denis, M., 2001. When and Why Are Visual Landmarks Used in Giving Directions? In: Montello, D. R. (Ed.), Spatial Information Theory. Foundations of Geographic Information Science : International Conference, COSIT 2001 Morro Bay, CA, USA, September 19-23, 2001. Proceedings. Vol. 2205 of Lecture Notes in Computer Science. Springer-Verlag, Berlin, Heidelberg, pp. 292-305.

Newman, E. L., Caplan, J. B., Kirschen, M. P., Korolev, I. O., Sekuler, R., Kahana, M. J., forthcoming 2006. Learning Your Way Around Town: How Virtual Taxicab Drivers Learn to use Both Layout and Landmark Information. Cognition .

Newman, M. E. J., 2005. Power Laws, Pareto Distributions and Zipf's Law. Contemporary Physics 46 (5), 323-351.

Newman, M. E. J., Girvan, M., 2004. Finding and Evaluating Community Structure in Networks. Physical Review E 69 (026113), 1-6.

Plumert, J. M., Carswell, C., de Vet, K., Ihrig, D., 1995. The Content and Organization of Communication about Object Locations. Journal of Memory and Language 37, 477-498.

Plumert, J. M., Spalding, T. L., Nichols-Whitehead, P., 2001. Preferences for As- 
cending and Descending Hierarchical Organization in Spatial Communication. Memory and Cognition 29 (2), 274-284.

Porta, S., Crucitti, P., Latora, V., 2006. The Network Analysis of Urban Streets: A Dual Approach. Physica A 369 (2), 853-866.

Raubal, M., Winter, S., 2002. Enriching Wayfinding Instructions With Local Landmarks. In: Egenhofer, M. J., Mark, D. M. (Eds.), Geographic Information Science: Second International Conference, GIScience 2002, Boulder, CO, USA, September 25-28, 2002. Proceedings. Vol. 2478 of Lecture Notes in Computer Science. Springer-Verlag, Berlin, Heidelberg, pp. 243-259.

Richter, K.-F., Klippel, A., Freksa, C., 2004. Shortest, Fastest, - but what's Next? A Different Approach to Route Directions. In: Muensteraner GI-Tage 2004. IfGIprints. Institut fuer Geoinformatik, Muenster, Germany.

Shanon, B., 1979. Where Questions. In: 17th Annual Meeting of the Association for Computational Linguistics. ACL, University of California at San Diego, La Jolla, California, USA.

Siegel, A. W., White, S. H., 1975. The Development of Spatial Representations of Large-Scale Environments. Advances in child development and behavior 10, 9-55.

Sorrows, M. E., Hirtle, S., 1999. The Nature of Landmarks for Real and Electronic Spaces. In: Freksa, C., Mark, D. (Eds.), Spatial Information Theory. Cognitive and Computational Foundations of Geographic Information Science: International Conference COSIT'99, Stade, Germany, August 1999. Proceedings. Vol. 1661 of Lecture Notes in Computer Science. Springer-Verlag, Berlin, Heidelberg, pp. 37-50.

Sperber, D., Wilson, D., 1982. Mutual Knowledge and Relevance in Theories of Comprehension. In: Smith, N. V. (Ed.), Mutual Knowledge. Academic Press, London, pp. 61-85.

Sperber, D., Wilson, D., 1986. Relevance. Basil Blackwell Ltd, Oxford, UK.

Stevens, A., Coupe, P., 1978. Distortions in Dudged Spatial Relations. Cognitive Psychology 10, 422-437.

Thomson, R. C., Richardson, D. E., 1999. The Good Continuation Principle of Perceptual Organization Applied to the Generalization of Road Networks. In: ICA 19th International Cartographic Conference. Ottawa, Canada.

Timpf, S., Kuhn, W., 2003. Granularity Transformations in Wayfinding. In: Freksa, C., Habel, C., Brauer, W., Wender, K. (Eds.), Spatial Cognition III - Routes and Navigation, Human Memory and Learning, Spatial Representation and Spatial Learning. Vol. 2685 of Lecture Notes in Computer Science. Springer-Verlag, Berlin, Heidelberg, pp. 77-88.

Timpf, S., Volta, G., Pollock, D., Egenhofer, M. J., 1992. A Conceptual Model of Wayfinding Using Multiple Levels of Abstraction. In: Frank, A., Campari, I., Formentini, U. (Eds.), Theories and Methods of Spatio-Temporal Reasoning in Geographic Space. International Conference GIS From Space to Territory: Theories and Methods of Spatio-Temporal Reasoning Pisa, Italy, September 2123, 1992 Proceedings. Vol. 639 of Lecture Notes in Computer Science. SpringerVerlag, Berlin, Heidleberg, pp. 348-367. 
Tomko, M., Winter, S., 2006a. Initial Entity Identification for Granular Route Directions. In: Kainz, W., Riedl, A., Elmes, G. (Eds.), Progress in Spatial Data Handling. 12th International Symposium on Spatial Data Handling. Springer-Verlag, Vienna, Austria, pp. 43-60.

Tomko, M., Winter, S., 2006b. Recursive Construction of Granular Route Directions. Journal of Spatial Science 51 (1), 101-115.

Tversky, B., 1993. Cognitive Maps, Cognitive Collages, and Spatial Mental Models. In: Frank, A., Campari, I. (Eds.), Spatial Information Theory A Theoretical Basis for GIS. European Conference, COSIT'93 Marciana Marina, Elba Island, Italy September 1922, 1993 Proceedings. Vol. 716 of Lecture Notes in Computer Science. Springer-Verlag, Berlin, Heidelberg, pp. 14-24.

Wiener, J. M., Mallot, H. A., 2003. 'Fine-to-Coarse' Route Planning and Navigation in Regionalized Environments. Spatial Cognition and Computation 3 (4), 331-358. 